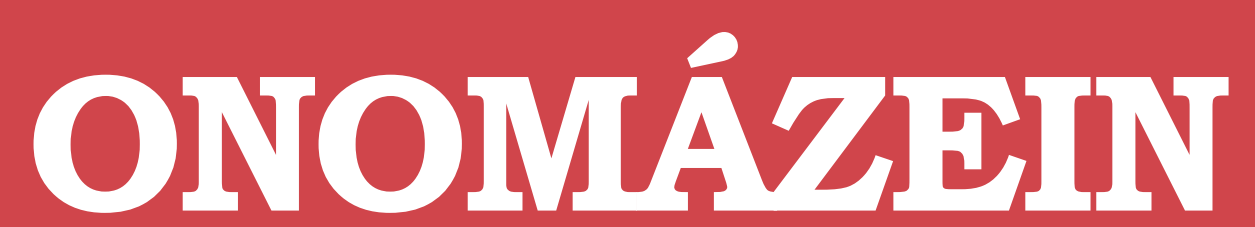

Revista semestral de lingüística, filología y traducción
PONTIFICIA UNIVERSIDAD CATÓLICA DE CHILE CATOLICA DE CHILE
FACULTAD DE LETRAS

\title{
Participantes y proyección en los procesos verbales en español: un análisis sistémico de géneros académicos estudiantiles
}

Participants and projection in verbal processes

in Spanish: a systemic analysis of academic

student genres

\author{
Natalia Ignatieva \\ Universidad Nacional Autónoma de México \\ México
}

\section{(c) $(i)$}

Natalia Ignatieva: Departamento de Lingüística Aplicada, Centro de Enseñanza de Lenguas Extranjeras, Universidad Nacional Autónoma de México. CELE, UNAM, C. U., Coyoacán, 04510, México, D. F.

Correo electrónico: ignatiev@unam.mx 


\section{Resumen}

El objetivo de este artículo es presentar un estudio sistémico funcional de procesos verbales, de los participantes asociados y de las características proyectantes de estos procesos en el lenguaje académico. El trabajo realizado forma parte de un proyecto de investigación que se ha desarrollado en la Universidad Nacional Autónoma de México (UNAM) y que, a su vez, está incluido en el proyecto internacional Systemics Across Languages (SAL). Los textos analizados fueron recopilados en la Facultad de Filosofía y Letras de la UNAM y forman parte del Corpus de Lenguaje Académico en Español (CLAE, 2009). Para el análisis que aquí se presenta se tomaron del CLAE textos pertenecientes a un área (literatura) y tres géneros distintos: pregunta-respuesta, ensayo y reseña. Las bases teóricas de este estudio se sitúan dentro de la perspectiva sistémico funcional (Halliday, 2004), particularmente, en el marco conceptual de la transitividad.
En primer lugar, se determina la frecuencia de los procesos verbales en el corpus y se identifican los participantes en distintos tipos de cláusulas verbales. Después, se explora con más detalle el participante más importante de la cláusula verbal, el Emisor. Se da también un énfasis especial al participante que expresa el mensaje del acto verbal, es decir, el Informe. Asimismo se examinan las características proyectantes de los procesos más usados en los textos escritos por estudiantes universitarios. Los resultados del estudio muestran que existe una variación importante entre los porcentajes de procesos verbales en cada uno de los géneros analizados. Por otra parte, diferentes formas de expresar "quién dice" y "qué dice" también contribuyen a las distinciones genéricas de los textos estudiantiles.

Palabras clave: Emisor; Informe; mensaje; género; proyectante.

\section{Abstract}

The aim of this paper is to present a systemic functional analysis of verbal processes, the associated participants and their projecting characteristics in academic language. This work forms part of the research study developed at the National Autonomous University of Mexico (UNAM), which, in turn, is included in the international project Systemics Across Languages (SAL). The analyzed student texts were collected at the Faculty of Arts of the UNAM and they form part of the Corpus of Academic Language in Spanish (CLAE, 2009). The texts that were taken for the present analysis belong to one area (literature) and three distinct genres: question-answer, essay and review. The theoretical bases of this study are rooted in a systemic functional perspective (Halliday, 2004), particularly, in the conceptual framework of transitivity.

The analysis begins with the calculation of fre- quency of verbal processes in our corpus. This, in turn, is followed by the identification of participants in the distinct types of verbal clauses. First, verbal process frequency in our corpus is determined and participants in distinct types of verbal clauses are identified. Then the most important participant in the verbal clause (Sayer) is explored in more detail. A special emphasis is also given to the participant expressing a message of the verbal act, i.e. the Verbiage. Similarly, projecting characteristics of the most frequent processes in the student texts are examined. The results of the study show that there is a significant variation among the verbal process percentages in each genre under analysis. On the other hand, different forms of expressing "who says" and "what is said" contribute to generic distinctions of the student texts as well. 


\section{Introducción}

En este artículo presentamos los resultados del proyecto centrado en los procesos verbales que aparecen en textos académicos escritos por estudiantes universitarios. Este trabajo forma parte de un proyecto que se lleva a cabo en la Universidad Nacional Autónoma de México ${ }^{1}$ y que es, a su vez, parte del proyecto internacional Systemics Across Languages (SAL), cuya versión latinoamericana es coordinada por Leila Barbara (Brasil). El propósito del proyecto SAL es explorar la "gramática" y los "usos discursivos" de cláusulas verbales y producir análisis sistémicos de varios registros y diferentes tipos de textos. Nuestro proyecto se inserta en esta tarea, y los resultados de la investigación realizada se reflejan en varias publicaciones (Ignatieva, 2011; Ignatieva y Zamudio, 2012; Ignatieva, en prensa). Los textos analizados fueron tomados de un proyecto anterior ${ }^{2}$, cuyo principal objetivo era estudiar el lenguaje de las humanidades en México y en los Estados Unidos (Ignatieva y Colombi, 2014). Una de las metas de dicho proyecto era la obtención de textos elaborados por estudiantes universitarios en el área de las humanidades, que ahora forman parte del Corpus de Lenguaje Académico en Español (CLAE, 2009)3. De este modo, para el análisis que aquí se presenta se tomaron del CLAE textos pertenecientes a tres géneros distintos, ensayos, reseñas y textos de pregunta-respuesta, elaborados por estudiantes mexicanos de la licenciatura en letras hispánicas.

En este artículo, primero, se va a definir el grupo de procesos verbales encontrados en estos textos, se determinará su frecuencia y se analizará su contexto de uso para así identificar a los participantes en las cláusulas verbales. Posteriormente, se examinarán algunos participantes del acto verbal, para determinar "quién dice qué” en cláusulas verbales. De esta manera, se va a efectuar un análisis más delicado del participante más importante, el Emisor, así como también de participantes que expresan el mensaje del acto verbal. Asimismo se examinarán las características proyectantes de los procesos más frecuentes en los textos estudiantiles. Antes de dar a conocer los resultados, hablaremos brevemente de la perspectiva teórica en la que se basa el proyecto.

\section{Fundamentos teóricos}

Este estudio escoge como su base la Lingüística Sistémico Funcional, propuesta por Halliday (1978, 1985, 2004), desarrollada posteriormente por otros investigadores dentro del área (Martin, 1985, 1992; Matthiessen, 1995; Thompson, 2004) y aplicada al español, entre otras lenguas (Ghio y Fernández, 2008; Montemayor-Borsinger, 2009; Lavid, Arús y Zamorano-Mansilla, 2010, entre otros). Esta teoría considera el lenguaje como una red de sistemas, formados a su vez por subsistemas que se basan en funciones, que se derivan del uso que se da del lenguaje dentro de un contexto social.

El lenguaje, en el pensamiento hallidayano (Halliday, 2004), se estructura de manera tal que cumple tres funciones principales, las llamadas metafunciones, que son la metafunción ideacional, la metafunción interpersonal y la metafunción textual. La metafunción ideacional organiza nuestras experiencias tanto en el mundo exterior como en el interior, la metafunción interpersonal expresa nuestra interacción con los

1 El proyecto, titulado Procesos verbales en la escritura académica a la luz de la Gramática Sistémico Funcional, se ha desarrollado en el Departamento de Lingüística Aplicada (Centro de Enseñanza de Lenguas Extranjeras). En él participan Natalia Ignatieva (coordinadora), Victoria Zamudio, Eleonora Filice, Daniel Rodríguez y Luz Elena Herrero.

2 El proyecto El lenguaje de las humanidades en México y los Estados Unidos: un análisis sistémico funcional se realizó en 2007-2010 como trabajo conjunto de dos equipos: de la Universidad Nacional Autónoma de México y de la Universidad de California, Davis

3 Este corpus se encuentra disponible en la página de internet http://lenguajeacademico.info. 
demás, mientras que la textual tiene como tarea contextualizar las unidades lingüísticas y organizarlas dentro de un discurso. De acuerdo con la perspectiva sistémica, estas tres metafunciones corresponden a tres sistemas dentro del nivel lexicogramatical: el sistema de transitividad, el de modo y el de tema. El sistema de transitividad es la realización de la función ideacional, el sistema de modo es reflejo de la función interpersonal y el de tema representa la función textual. El presente estudio se ubica dentro del sistema de transitividad explorando el significado experiencial de la metafunción ideacional del lenguaje.

Como dice Halliday (1976: 21), "la transitividad es el componente ideacional en las cláusulas”, en términos del potencial de significado. Este sistema construye gramaticalmente la experiencia a partir de diversos tipos de procesos que se pueden realizar. A cada tipo de proceso en la cláusula le corresponden diferentes combinaciones de participantes, las cuales permiten representar todo tipo de experiencias en una lengua dada y con ello ordenar la diversidad de eventos que ocurren de manera constante (Halliday, 2004).

El sistema de transitividad es, entonces, el que permite construir y organizar las diversas experiencias. Es necesario señalar que, originalmente, Halliday (1968) establece tres tipos de procesos: Ios "materiales", los "mentales" y los "relacionales". Más adelante, a estos les suma tres tipos más: los procesos "verbales", los procesos "conductuales" y los "existenciales" (Halliday, 1985). Los tres primeros son considerados procesos básicos mientras que los tres posteriores son vistos como procesos intermedios o secundarios.

Los procesos verbales, como procesos intermedios, se sitúan entre los procesos mentales y los relacionales puesto que una relación simbólica, que se construye en la conciencia humana, se manifiesta en el lenguaje como el decir (Halliday, 2004). Cabe señalar, sin embargo, que Matthiessen (1995) considera que son cuatro los tipos básicos de procesos. A los tres tipos propuestos por Halliday (materiales, mentales y relacionales) Matthiessen añade los verbales como otro proceso básico, ya que, apunta, estos tienen características propias que los diferencian y separan de otros tipos de procesos. En el presente estudio hemos seguido el punto de vista propuesto por Matthiessen.

Cabe aclarar que las categorías de los tipos básicos de procesos fueron propuestas por Halliday para el inglés. Sin embargo, Halliday opina que su clasificación de tipos de procesos refleja ciertos principios semánticos universales y es aplicable a otras lenguas (2004); esto ya fue comprobado en algunos trabajos que aplicaron esta clasificación para la descripción sistémica del español (Ghio y Fernández, 2008; Lavid, Arús y Zamorano-Mansilla, 2010)4.

Como mencionamos arriba, cada tipo de proceso conlleva diferentes participantes, formando así su propio modelo - o esquema - con el que construye el campo de experiencia que le compete. Dicho modelo incluye el papel que juegan los diferentes participantes en las cláusulas donde aparece un tipo particular de proceso (Lavid, Arús y Zamorano-Mansilla, 2010). De esta manera, los procesos verbales tienen su propio esquema para construir la cláusula, del cual hablaremos brevemente a continuación.

Creemos que es importante señalar que en nuestro análisis tratamos de tomar en cuenta todos los verbos que expresan el proceso de decir. En esto seguimos la tendencia general dentro del proyecto SAL de adoptar un punto de vista lo más incluyente posible, pero a la vez de acuerdo con Halliday (2004). Por ello registramos todos los verbos que se usaron para referirse a un acto de habla.

4 Cabe recordar que el proyecto SAL tiene un carácter tipológico, y una de sus metas es explorar cómo se realizan las categorías sistémico funcionales en diferentes lenguas. 


\subsection{Los participantes y proyección en las cláusulas verbales}

De acuerdo con Halliday, los participantes más comunes en una cláusula verbal son Emisor (quién dice), Receptor (a quién se dirige el mensaje) e Informe (qué se dice); por ejemplo, en (1) Don Quijote sería el Emisor; le, el Receptor, y su locura, el Informe. Además, Halliday subraya que el único participante realmente necesario para el acto de decir es el Emisor.

(1) Don Quijote le cuenta su locura... (E, 4) ${ }^{5}$

Otro participante relacionado con el mensaje del proceso verbal es el Asunto, que a diferencia del Informe se realiza por una frase preposicional como sobre etimología en (2):

(2) Gómez habla sobre etimología,... $(R, 2)$

De los participantes mencionados, solamente el Emisor es considerado por Halliday como participante "directo", mientras que el Receptor y el Informe son vistos como participantes "oblicuos", el Asunto siendo el "equivalente circunstancial del Informe” (Halliday, 2004: 276).

Otra manera de transmitir el mensaje es representarlo a partir del discurso directo o indirecto. En el ejemplo (3) podemos ver que se presentan dos cláusulas y, de ellas, solo la primera es verbal. La segunda es una cláusula dependiente proyectada, comúnmente analizada como "cláusula reportada", siendo la relación entre ambas una relación hipotáctica. Del mismo modo, en el ejemplo (4) vemos también un complejo de cláusulas; sin embargo, la relación entre ellas es paratáctica, es decir, las dos cláusulas son independientes; además, la segunda cláusula presenta una cita directa.

(3) Carreira señala que el artista debe sentirse libre... $(R, 7)$
(4) ... y le cuenta su tristeza: “¿Dónde estás señora mía,... ?’ (P-R, 11)

En estos casos, la cláusula proyectada que contiene el mensaje es Ilamada "locución" (Iocution) directa o indirecta, pero las locuciones no son participantes porque se encuentran fuera de la cláusula verbal. Cuando el mensaje se expresa por medio de lenguaje directo o indirecto, hay una relación lógico-semántica entre el miembro primario y el miembro secundario del complejo de cláusulas. En la gramática sistémico funcional a esta relación se le denomina "proyección" (Halliday, 2004). Thompson (2004: 103) describe esta relación comparándola con una pintura (que sería la cláusula proyectada) y su marco (la cláusula que hace la proyección), en donde los dos elementos forman una unidad pero ninguno es parte del otro.

De acuerdo con lo dicho anteriormente, los procesos verbales se dividen en dos grupos: proyectantes que pueden tener cláusulas proyectadas, como (3) y (4), y no proyectantes cuando no las tienen, como (1) y (2). Sería importante mencionar que no todos los lingüistas consideran estos últimos como procesos verbales (Martin, Matthiessen y Painter, 1997), ya que toman la proyección como criterio para distinguir entre procesos verbales (que proyectan) y otros tipos de procesos de comunicación (que no proyectan). En este caso los procesos no proyectantes se excluyen del grupo de procesos verbales y se definen como procesos conductuales. Los Ilaman también "procesos de conducta verbal", ya que procesos como hablar o platicar están en la frontera entre los procesos verbales y los conductuales (Matthiessen, 1995: 284). Banks, sin embargo, señala que no hay frontera entre los procesos verbales y conductuales, de acuerdo al diagrama de Halliday en la portada de la segun-

5 Todos los ejemplos están tomados de los textos estudiantiles de nuestro corpus; se conserva la ortografía de los alumnos.

6 Matthiessen, empero, no es tan categórico cuando dice que "estos verbos pueden ser interpretados como conductuales" (énfasis mío); en otras palabras, él admite otras posibilidades de análisis (1995: 284). 
da edición de Introduction to Functional Grammar (es decir, estos dos tipos de procesos no son adyacentes), por lo cual la postura mencionada arriba resulta contradictoria (Banks, en prensa). En nuestro proyecto, consideramos ambos grupos como verbales siguiendo a Caffarel (2006: 8688) y Banks (en prensa) y tomando en cuenta que los dos tipos de procesos participan en el acto de comunicación

Consecuentemente, en la figura 1 mostramos los componentes que conforman las tres partes de nuestro análisis: Ios procesos verbales, los participantes en las cláusulas verbales y las locuciones (proyecciones).

\section{FIGURA 1}

Los componentes del análisis de participantes y proyecciones

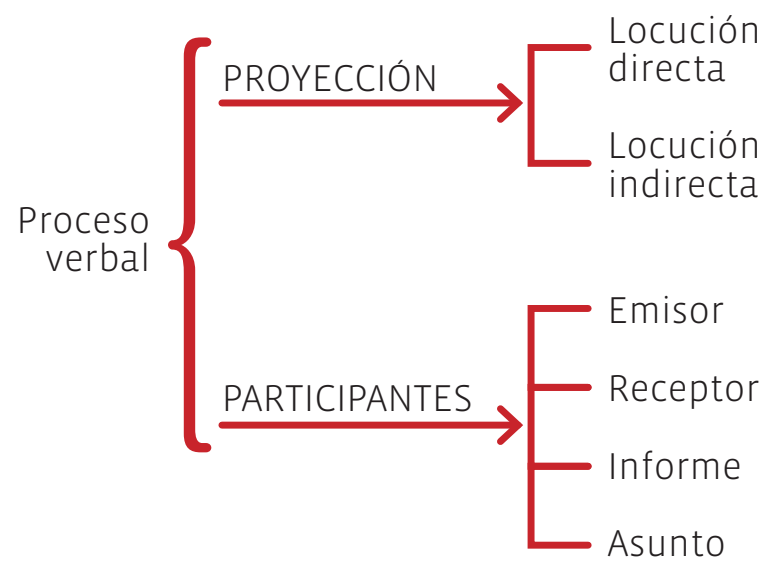

\section{Metodología}

Entre los objetivos de nuestro estudio están: definir el grupo de los procesos verbales, determinar su frecuencia, identificar los participantes de cláusulas verbales y explorar las características proyectantes de los procesos más frecuentes.
Como ya mencionamos, utilizamos el corpus CLAE, esto es, tomamos de él textos estudiantiles recolectados en la Facultad de Filosofía y Letras de la Universidad Nacional Autónoma de México (UNAM). Este artículo está basado en el análisis de textos de literatura. Son 31 textos, todos escritos por los alumnos del Colegio de Letras Hispánicas, del segundo semestre. Los textos pertenecen a tres géneros ${ }^{8}$ : pregunta-respuesta (15), ensayo (7) y reseña (9). El primer género es pregunta-respuesta $(\mathrm{P}-\mathrm{R})$, que constituye parte del examen escrito en clase y es un texto corto con extensión máxima de una cuartilla. El segundo es un ensayo (E) más largo preparado por los alumnos fuera del aula como trabajo semestral; su extensión varía de 5 a 20 cuartillas. Y el tercero, reseña $(R)$, es un trabajo también escrito fuera del aula, con extensión de 3 a 5 páginas.

Para el estudio del corpus se va a realizar un análisis cuantitativo que permite conocer la proporción y frecuencia en la utilización de los verbos y, consecuentemente, detectar las opciones que hacen lo estudiantes en su escritura. Asimismo, un análisis de tipo más cualitativo nos dará la oportunidad de explorar su contexto de uso y sus rasgos discursivos. Como el corpus explorado es relativamente pequeño, el análisis cuantitativo se realiza manualmente.

Asimismo se va a efectuar un análisis contrastivo entre los géneros explorados.

\section{Procesos verbales en nuestro corpus}

Para definir los procesos verbales utilizamos básicamente el criterio semántico, el mismo que usó Halliday para construir su sistema de transitividad. Consideramos como procesos verbales todos los verbos que tienen que ver con el proceso de decir y participan en el acto de comu-

7 Por otra parte, Banks critica la categoría de procesos conductuales por ser una categoría híbrida, no definida con criterios claros, y propone descartarla por completo, ya que, en su opinión, todos los subtipos (heterogéneos) de procesos que están allí podrían ser ubicados dentro de otras categorías semánticas.

8 No se profundiza más sobre el tema de género en este trabajo porque este término, al igual que los nombres de los géneros particulares (ensayo, reseña, pregunta-respuesta), se utiliza como término operativo. A partir de los datos, aplicamos las etiquetas que se usan en el ambiente universitario para referirse a estos textos. 
nicación (verbos dicendi)9. Empezamos nuestro análisis considerando algunos datos cuantitativos de los procesos verbales en nuestro corpus; estos datos se presentan en la tabla 1.

\section{TABLA 1}

Comparación de los datos de la frecuencia verbal en los textos P-R, E y R

\begin{tabular}{c|c|c|c} 
& CLÁUSULAS & $\begin{array}{c}\text { PROCESOS } \\
\text { VERBALES }\end{array}$ & $\%$ PV \\
\hline $\begin{array}{c}\text { Pregunta- } \\
\text { respuesta }\end{array}$ & 427 & 108 & 25.3 \\
\hline Ensayo & 900 & 102 & 11.3 \\
\hline Reseña & 565 & 160 & 28.3
\end{tabular}

Se puede ver que en los textos del tipo pregunta-respuesta el número total de cláusulas es 427 con 108 casos de procesos verbales, lo cual parece un número bastante grande, que corresponde a $25.3 \%$ en relación al número de cláusulas $^{10}$. Los ensayos presentan un cuadro diferente: tienen un mayor número de cláusulas (900), pero la cantidad de procesos verbales disminuye (102), lo que corresponde a $11.3 \%$. El tercer género, reseñas, sobrepasa los otros dos en la cantidad de procesos verbales (160) y su porcentaje (28.3\%), aunque no en el número de cláusulas (565). Entonces, se puede apreciar en la figura 2

\section{FIGURA 2}

Comparación de los procesos verbales en los tres géneros

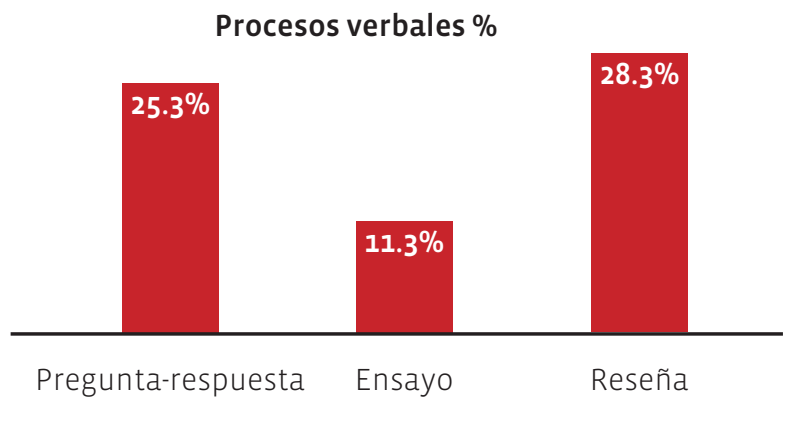

que existe cierta similitud entre los textos P-R y $\mathrm{R}$ mientras que los ensayos difieren de los otros géneros en este sentido.

El siguiente paso fue ver los resultados del análisis de participantes y proyección (APP) que fue realizado en el proyecto (Ignatieva, en prensa). Los resultados de este análisis se aprecian en la tabla 2.

Los datos del APP resultan bastante heterogéneos. El único participante que tiene valores parecidos en los tres géneros es el Asunto; los demás tienen más diferencias que similitudes. Sin embargo, el participante que se realiza con mayor frecuencia en cada género (con excepción de la reseña) es el Emisor, del cual ofrecemos un análisis más detallado a continuación.

\section{TABLA 2}

Datos resumidos del APP de los tres géneros

\begin{tabular}{c|c|c|c|c|c|c|c} 
Textos & $\begin{array}{c}\text { Emisor } \\
\%\end{array}$ & $\begin{array}{c}\text { Proceso } \\
\%\end{array}$ & $\begin{array}{c}\text { Receptor } \\
\%\end{array}$ & $\begin{array}{c}\text { Asunto } \\
\%\end{array}$ & $\begin{array}{c}\text { Informe } \\
\%\end{array}$ & $\begin{array}{c}\text { Locución } \\
\text { indirecta } \\
\%\end{array}$ & $\begin{array}{c}\text { Locución } \\
\text { directa } \\
\%\end{array}$ \\
\hline P-R & 53.7 & 100 & 32.4 & 18.5 & 37 & 42.6 & 1.85 \\
\hline$E$ & 42.57 & 100 & 40.6 & 25.7 & 36.6 & 26.7 & 14.8 \\
\hline$R$ & 42.15 & 100 & 5.6 & 21.87 & 53.75 & 13.12 & 6.25
\end{tabular}

9 Cabe mencionar que los verbos dicendi (como decir, hablar, conversar, etc.) son ampliamente citados en la literatura lingüística como una clase semántica bien identificada (véase, por ejemplo, Van Valin y LaPolla, 1997; Ibáñez, 2008, entre otros).

10 Se contaron tanto cláusulas en rango como cláusulas incrustadas. 


\section{Análisis de emisores}

Pasamos, entonces, al análisis del participante más importante de la cláusula verbal, de acuerdo con Halliday, el Emisor, para explorar "quién habla". La tabla 3 nos repite los datos de la tabla 2 en relación a los emisores de nuestro corpus; cabe mencionar que estos datos solo incluyen los emisores expresados explícitamente en el texto. Llama la atención que el número de cláusulas donde el emisor se realiza explícitamente constituye aproximadamente la mitad del corpus. En cuanto a su relación con el género del texto, se puede ver que el número de emisores explícitos es mayor en los textos P-R (53.7\%) y disminuye en los ensayos (42.57\%) y reseñas (42.13\%). Estos dos últimos géneros tienen un número muy parecido.

\section{TABLA 3}

Datos de emisores en los tres géneros

\begin{tabular}{c|c|c|c} 
& $\begin{array}{c}\text { Pregunta- } \\
\text { respuesta }\end{array}$ & Ensayo & Reseña \\
\hline Emisores & $53.7 \%$ & $42.57 \%$ & $42.13 \%$
\end{tabular}

\subsection{Emisores explícitos, implícitos 0 ausentes}

A continuación, nos propusimos hacer un análisis más delicado, y con este fin dividimos las cláusulas verbales en tres grandes grupos de acuerdo con la realización (o no realización) del emisor y con el tipo de emisor no expresado:

1) cláusulas con emisores explícitos,

2) cláusulas con emisores implícitos,

3) cláusulas con emisores ausentes (cláusulas impersonales o no finitas).

De esta manera, en el primer grupo están todos los emisores realizados explícitamente en el texto (ver ej. 5); en el segundo, los emisores implícitos, cuando el emisor se omite porque se ha mencionado antes y/o se entiende por el contexto (ej. 6), y en el tercer grupo están los emisores ausentes, cuando las cláusulas son impersonales (ej. 7) o no finitas, es decir, expresadas por un infinitivo, gerundio o participio (ej. 8).

(5) Diez señala la problemática popular en el patio de la presentación. $(R, 5)$

(6) Cito a Erasmo de Rotterdam... (E, 6)

(7) Se menciona el trabajo de las Anotaciones. $(R, 9)$

(8)Argumentando que para profesarla se ha de saber de leyes... (P-R, 5)

La tabla 4 presenta los datos de estos tres tipos de emisores en nuestro corpus en relación a los tres géneros explorados.

\section{TABLA 4}

Porcentaje de emisores explícitos o implícitos

\begin{tabular}{l|c|c|c} 
& $\begin{array}{c}\text { Pregunta- } \\
\text { respuesta } \\
\text { (108 PV) }\end{array}$ & Ensayo & Reseña \\
\hline (101 PV) & (159 PV) \\
\hline Emisores explícitos & $53.70 \%$ & $42.57 \%$ & $42.13 \%$ \\
\hline Emisores implícitos & $31.48 \%$ & $26.73 \%$ & $26.41 \%$ \\
\hline Emisores ausentes & $\mathbf{1 4 . 8 1 \%}$ & $30.69 \%$ & $31.44 \%$
\end{tabular}

Se puede apreciar que de los tres tipos de emisores los que prevalecen en nuestro corpus son los explícitos. En cuanto a los emisores implícitos, su cantidad es más grande en los textos de pregunta-respuesta, en comparación con los otros dos géneros ${ }^{11}$. En cambio, el número de sujetos no realizados en cláusulas impersonales o no finitas es mayor en los ensayos y reseñas comparados con las preguntas-respuestas. Estas características se deben, tal vez, a las condiciones de su elaboración, pues mientras que los textos P-R son redactados en clase a manera de examen, los textos E y R son hechos fuera de aula con mayor tiempo para realizarse, de ahí el carácter menos formal del género pregunta-

11 Aunque la omisión del sujeto es habitual en español, la cantidad de sujetos omitidos disminuye en el lenguaje académico de autores expertos en comparación con el lenguaje académico estudiantil, que muestra marcas de oralidad, de acuerdo con el estudio realizado por Filice (2008). 
respuesta, donde se encuentran más marcas del lenguaje oral (Ignatieva, 2008). En contraste con esto, los textos de los otros dos géneros (ensayo y reseña) utilizan un lenguaje que se acerca mucho más al estilo escrito, de ahí la cantidad más grande de cláusulas impersonales y no finitas, que son características del medio escrito.

\section{Análisis del mensaje y las formas de expresarlo}

El siguiente paso es ver "qué se dice" en las cláusulas verbales. La tabla 5 nos proporciona los datos del análisis de participantes y proyección (APP) que realizamos dentro del proyecto (Ignatieva, en prensa); en esta tabla están incluidos solamente los participantes que expresan el contenido del mensaje (Informe y Asunto) y las cláusulas proyectadas (locución directa e indirecta).

Como se puede apreciar en la tabla 5, el participante predominante en general resulta ser el Informe, mientras que la locución indirecta se destaca como la forma preferida de realizar el mensaje en el género de pregunta-respuesta.

\subsection{Procesos proyectantes y no proyectantes}

De acuerdo con el criterio de proyección mencionado en el marco teórico, dividimos los procesos verbales en dos grupos grandes: proyectantes y no proyectantes. La tabla 6 muestra los valores numéricos de esta división. Llama la atención el hecho de que el número de las cláusulas no proyectantes en total dobla el de las cláusulas proyectantes, lo cual pone de manifiesto las opciones de los alumnos cuando ellos describen situaciones de comunicación.

\section{TABLA 6}

Cláusulas proyectantes y no proyectantes

\begin{tabular}{c|c|c|c|c}
\multirow{2}{*}{} & \multicolumn{2}{|c|}{$\begin{array}{c}\text { Cláusulas } \\
\text { proyectantes }\end{array}$} & \multicolumn{2}{c}{$\begin{array}{c}\text { Cláusulas no } \\
\text { proyectantes }\end{array}$} \\
\cline { 2 - 5 } & Número & $\%$ & Número & $\%$ \\
\hline Textos P-R & 48 & $\mathbf{4 4 . 4}$ & 60 & $\mathbf{5 5 . 6}$ \\
\hline Textos E & 42 & $\mathbf{4 1 . 6 \%}$ & 59 & $\mathbf{5 8 . 4 \%}$ \\
\hline Textos R & 31 & $\mathbf{1 9 . 4 \%}$ & 129 & $\mathbf{8 0 . 6 \%}$ \\
\hline Total & $\mathbf{1 2 1}$ & $\mathbf{3 2 . 8 \%}$ & $\mathbf{2 4 8}$ & $\mathbf{6 7 . 2} \%$
\end{tabular}

Por otra parte, los textos P-R y E demuestran un cuadro parecido en cuanto a la cantidad de las cláusulas proyectantes y no proyectantes, mientras que los textos $\mathrm{R}$ difieren de los otros géneros en relación a este parámetro.

\subsubsection{Cláusulas proyectantes}

Entre las cláusulas proyectantes se nota que los estudiantes utilizan con más frecuencia locuciones indirectas, es decir, prefieren reportar en vez de citar y, consecuentemente, usar hipotaxis (ejs. 9 y 10) en vez de parataxis (ej. 11):

(9) Él afirma que nadie tienen que porqué hablar mal de ella. $(P-R, 13)$

(10) En el primer capítulo, José María Micó advierte que no hay poema conservado de poeta antes de 1580. $(R, 6)$

(11) ... a lo que el responde: "Venid acá, gente soez y mal nacida..." $(\mathrm{E}, 7)$

\section{TABLA 5}

Datos del APP para los tres géneros

\begin{tabular}{c|c|c|c|c|c} 
Textos & $\begin{array}{c}\text { Proceso } \\
\%\end{array}$ & $\begin{array}{c}\text { Asunto } \\
\%\end{array}$ & $\begin{array}{c}\text { Informe } \\
\%\end{array}$ & $\begin{array}{c}\text { Locución } \\
\text { indirecta } \\
\%\end{array}$ & $\begin{array}{c}\text { Locución } \\
\text { directa } \\
\%\end{array}$ \\
\hline P-R & 100 & 18.5 & 37 & 42.6 & 1.85 \\
\hline$E$ & 100 & 25.5 & 36.3 & 26.5 & 14.7 \\
\hline$R$ & 100 & 21.87 & 53.75 & 13.12 & 6.25
\end{tabular}


Si tomamos la totalidad de las cláusulas proyectantes por 100\%, entonces el porcentaje de casos de discurso indirecto llega a 95.8\% en preguntas-respuestas, a $64.3 \%$ en ensayos y a $67.7 \%$ en reseñas, mientras que los números correspondientes para el discurso directo son $4.2 \%, 35.7 \%$ y $32.3 \%$, respectivamente (tabla 7 ). Se puede deducir que los estudiantes básicamente usan citas en ensayos y reseñas y casi no lo hacen en preguntas-respuestas.

\subsubsection{Cláusulas no proyectantes}

En el grupo de procesos no proyectantes la verbalización se expresa con ayuda de dos participantes: Informe (ej. 12) y Asunto (ej. 13):

(12) Se menciona otro poema de Góngora (Ciego que apuntas $y$ atinas) que viene a ser la contraparte del anterior. (R. 6)

(13) Esta voz nos habla un poco del aspecto mítico del caballero. $(\mathrm{P}-\mathrm{R}, \mathrm{9})$
Como se ve en la tabla 8, el medio preferido para expresar el mensaje en los tres géneros es el Informe; aproximadamente dos terceras partes de las cláusulas no proyectantes realizan el contenido de mensaje por medio del Informe (65.7\% en promedio), mientras una tercera parte lo hace vía el Asunto, que es el subtipo del Informe.

\section{Características proyectantes de los procesos más frecuentes}

Como etapa final de nuestro estudio analizamos los procesos más frecuentes de nuestro corpus a la luz de sus capacidades proyectantes desde la escala de su frecuencia. La figura 3 muestra los primeros diez procesos.

Como se puede apreciar, el verbo decir sobrepasa ampliamente todos los demás procesos en cuanto a su frecuencia, siendo el proceso dominante del grupo. En el segundo y tercer lugar están los verbos mencionar y hablar, respectivamente.

\section{TABLA 7}

Cláusulas proyectantes

\begin{tabular}{c|c|c|c|c|c|c}
\multirow{2}{*}{} & \multicolumn{2}{|c|}{ Cláusulas } & \multicolumn{2}{c|}{$\begin{array}{c}\text { Locución } \\
\text { indirecta }\end{array}$} & \multicolumn{2}{c}{$\begin{array}{c}\text { Locución } \\
\text { directa }\end{array}$} \\
\cline { 2 - 7 } & Número & $\%$ & Número & $\%$ & Número & $\%$ \\
\hline Textos P-R & 48 & $100 \%$ & 46 & 95.8 & 2 & 4.2 \\
\hline Textos E & 42 & $100 \%$ & 27 & 64.3 & 15 & 35.7 \\
\hline Textos R & 31 & $100 \%$ & 21 & 67.7 & 10 & 32.3 \\
\hline Total & 121 & $100 \%$ & 94 & $\mathbf{7 7 . 7}$ & $\mathbf{2 7}$ & $\mathbf{2 2 . 3}$
\end{tabular}

\section{TABLA 8}

Cláusulas no proyectantes

\begin{tabular}{c|c|c|c|c|c|c} 
& \multicolumn{2}{|c|}{$\begin{array}{c}\text { Cláusulas no } \\
\text { proyectantes }\end{array}$} & \multicolumn{2}{c|}{ Asunto } & \multicolumn{2}{c}{ Informe } \\
\cline { 2 - 7 } & Número & $\%$ & Número & $\%$ & Número & $\%$ \\
\hline Textos P-R & 60 & $\mathbf{1 0 0 \%}$ & 20 & $\mathbf{3 3 . 3}$ & 40 & $\mathbf{6 6 . 7}$ \\
\hline Textos E & 59 & $\mathbf{1 0 0 \%}$ & 26 & $\mathbf{4 4}$ & 37 & $\mathbf{6 2 . 7}$ \\
\hline Textos R & 129 & $\mathbf{1 0 0 \%}$ & 35 & $\mathbf{2 7 . 1}$ & 86 & $\mathbf{6 6 . 7}$ \\
\hline Total & $\mathbf{2 4 8}$ & $\mathbf{1 0 0 \%}$ & $\mathbf{8 1}$ & $\mathbf{3 2 . 6}$ & $\mathbf{1 6 3}$ & $\mathbf{6 5 . 7}$
\end{tabular}




\section{FIGURA 3}

Diez procesos más frecuentes del corpus estudiantil

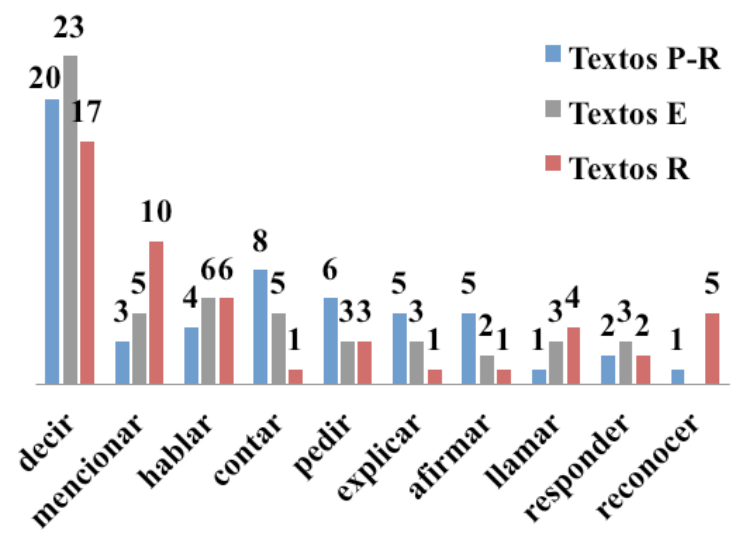

A continuación analizamos las características proyectantes de los diez procesos que se presentan en la figura 4.

\section{FIGURA 4}

Características proyectantes de los diez procesos más frecuentes

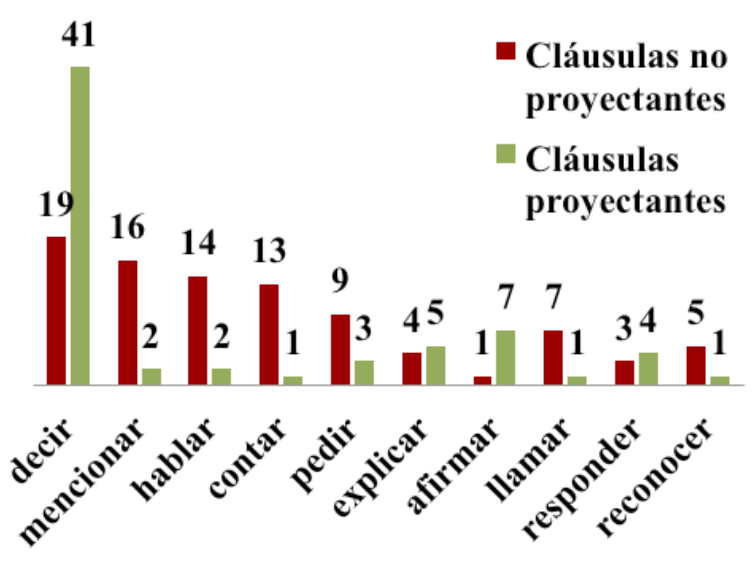

Los datos de la figura 4 sugieren que todos los procesos pueden tener "doble uso", ya que pueden ser tanto proyectantes como no proyectantes.

(14) ... la doncella le pide dinero a Don Quijote para Dulcinea. (P-R, 2)

(15) Le pide que corteje a Camila, cuya fidelidad desea poner a prueba. (E, 2)
Como se puede apreciar en los ejemplos arriba, el verbo pedir no es proyectante en el ejemplo (14) y requiere de un participante (dinero) que es Informe, mientras que en el ejemplo (15) el mismo verbo proyecta la cláusula que corteje a Camila. Se puede observar lo mismo en los ejemplos (16), uso no proyectante, y (17), uso proyectante, con el verbo decir:

(16) Sancho le decía lo contrario y así sucesivamente. $(\mathrm{P}-\mathrm{R}, 12)$

(17) ... debo decir que la lectura de este libro me ayudó a ampliar mi campo de visión... $(R, 3)$

Mientras que cuatro verbos (decir, explicar, afirmar y responder) tienen más estructuras proyectantes que no proyectantes, otros seis verbos prefieren estructuras no proyectantes ${ }^{12}$. En total, se encuentran más estructuras no proyectantes en los primeros diez procesos (91 sumando todas) en comparación con las proyectantes (67 en suma), lo cual, a su vez, concuerda con los datos de todo el corpus.

\section{Conclusiones}

En este artículo hemos analizado el uso de los procesos verbales en los escritos estudiantiles de literatura, pertenecientes a tres géneros: pregunta-respuesta, ensayo y reseña.

Antes que nada, queremos subrayar que la presencia de los procesos verbales en nuestro corpus es sustancial (21.63\%), lo cual quiere decir que los procesos verbales ocupan un lugar importante entre los recursos lingüísticos que escogen los estudiantes en sus actividades de escritura. Cabe recordar que en general la frecuencia de aparición de los elementos lingüísticos en el discurso es un buen índice de su importancia en el sistema lingüístico. Esto también comprueba la legitimidad de considerar los procesos verbales como uno de los tipos principales, al lado de los procesos materiales, relacionales y mentales, en el sistema de transitividad.

12 Es de notar que algunos verbos que generalmente no proyectan, como hablar, por ejemplo, se utilizan en el corpus estudiantil para introducir el discurso directo. 
En cuanto al análisis más fino de uno de los participantes más importantes del acto de hablar, el Emisor, este se manifiesta explícitamente casi en la mitad de las cláusulas verbales, siendo las preguntas-respuestas el género donde hay más emisores explícitos. Esto se explica por la preferencia de cláusulas finitas en los textos P-R en comparación con los textos $E$ y $R$, donde los estudiantes utilizan mayor cantidad de cláusulas impersonales o no finitas. Ya mencionamos antes que los textos P-R tienen marcas de oralidad y de esta manera contienen mayor complejidad gramatical (es decir, mayor número de cláusulas finitas). Al mismo tiempo, en los textos P-R sobresalen los emisores implícitos, comparándolos con los números de los otros dos géneros. Esto significa que los escritores omiten frecuentemente el sujeto de la cláusula (y, consecuentemente, el Emisor del proceso) acercando su escrito al lenguaje oral, ya que la frecuencia de sujetos omitidos en el lenguaje oral es mucho mayor que en el lenguaje escrito.

En relación a la transmisión del mensaje en la cláusula verbal, hemos podido observar que los alumnos utilizan diferentes opciones para este fin. En general, prevalecen los usos no proyectantes con el Informe como el medio preferido para expresar el contenido de la comunicación. Esta prevalencia apunta al importante papel que juegan los usos no proyectantes de procesos verbales en el discurso estudiantil. Entre los usos proyectantes se encuentran más las locuciones indirectas que las directas.

En nuestro análisis exploramos también los diez procesos más frecuentes del corpus y sus capacidades proyectantes. El verbo dominante de los tres subcorpus ha sido el verbo decir (aproximadamente $20 \%$, es decir, una quinta parte del total de los procesos verbales). Todos los verbos entre los primeros diez se utilizan tanto en las cláusulas proyectantes como en las no proyectantes. Esto, a su vez, puede sugerir que tanto la proyección como su ausencia (con el uso de otros recursos) son rasgos característicos de los procesos verbales. Tomando en cuenta el "do- ble uso" de los procesos verbales más frecuentes sería ilógico realizar la división de estos procesos en verbales y conductuales con el criterio de proyección.

El análisis de participantes y proyección nos ha permitido establecer algunas características de cada género y comparar los tres géneros estudiados. A la luz de diferentes parámetros explorados se pudo detectar que en general los géneros de ensayo y reseña tienen muchos rasgos en común mientras que el género de pregunta-respuesta difiere bastante de los otros dos. Esto en gran medida se debe a distintas condiciones de elaboración de estos escritos, como lo pudimos constatar. Se puede deducir que en términos generales los textos pregunta-respuesta se caracterizan por un estilo simple, informal y casi coloquial, mientras que los ensayos y las reseñas adquieren un carácter más elaborado y formal, al igual que más objetivo y abstracto, lo cual confirma nuestro estudio anterior (Ignatieva, 2008, 2010).

En resumen, el presente análisis de los procesos verbales en el lenguaje estudiantil nos dio la oportunidad de destacar algunas características discursivas de la escritura académica relacionadas con la expresión del acto de decir, así como las diferencias y similitudes entre los géneros analizados.

\section{Bibliografía citada}

BAnks, David, en prensa: "On the (non)necessity of the hybrid category behavioural process" en Donna R. Miller y Paul Bayley (eds.): Hybridity in systemic functional linguistics: Grammar, text and discursive context, Londres: Equinox.

Caffarel, Alice, 2006: A systemic functional grammar of French: From grammar to discourse, London: Continuum.

CLAE, 2009: El lenguaje académico en español. Análisis binacional de textos en las humanidades [http://www.lenguaje academico.info, fecha de consulta: 10 de septiembre de 2013]. 
FILICE, Eleonora, 2008: La estructura temática en los géneros académicos: Un análisis sistémico funcional de escritos de geografía a nivel universitario. Tesis de maestría. México: Universidad Nacional Autónoma de México.

Ghio, Elsa y Ma. Delia Fernández, 2008 [2006]: Manual de Lingüística Sistémico Funcional: el enfoque de M. A. K. Halliday y R. Hasan: aplicaciones a la lengua española, Santa Fe, Argentina: Universidad Nacional del Litoral.

Halliday, Michael A. K., 1968: "Notes on transitivity and theme in English", Journal of Linguistics 4, 179-215.

Halliday, Michael A. K., 1976: System and function in language, London: Oxford University Press

HallidaY, Michael A. K., 1978: Language as social semiotic: The social interpretation of language and meaning, London: Arnold.

Halliday, Michael A. K., 1985: Spoken and written language, Geelong, Vic.: Deakin University Press. Halliday, Michael A. K., 2004 [1994]: An introduction to functional grammar, London: Arnold.

IBÁÑEZ,Sergio, 2008:"SayingverbsinSpanish.Deepening the lexical semantics" en Robertvan Vauin (ed.): Investigations of the syntax-semantics-pragmatics interface, Amsterdam: John Benjamins, 3-21.

Ignatieva, Natalia, 2008: "Question-answer as a genre in students' academic writing in Spanish" en Canzhong Wu, Christian M. Matthiessen y Maria HeRke (comps.): Proceedings of ISFC 35: Voices around the World, Sydney: Macquarie University, 61-66.

Ignatieva, Natalia, 2010: "Ensayo como género de la escritura académica estudiantil en español en el marco de la lingüística sistémica funcional", Lingüística Mexicana 1, vol. V, 91-109.

Ignatieva, Natalia, 2011: "Verbal processes in student academic writing in Spanish from a systemic functional perspective", Lenguaje 2, vol. 39, 447-467.

Ignatieva, Natalia, en prensa: "Transitivity and genre: Verbal processes in student academic texts of three genres in Spanish" en Proceedings of the 38th ISFC, Lisboa.
Ignatieva, Natalia y Victoria Zamudio, 2012: "Perspectiva funcional de los procesos verbales en los escritos estudiantiles de literatura e historia en español”, DELTA (Documentação de Estudos em Lingüística Teórica e Aplicada), PUC-SP, Brazil, vol. 28, no. spe., 561-579.

Ignatieva, Natalia y Cecilia Colombi (coords.), 2014: CLAE: El lenguaje académico en México y los Estados Unidos: un análisis sistémico funcional, México: Universidad Nacional Autónoma de México.

Lavid, Julia, Jorge Arús y Juan R. Zamorano-Mansilla, 2010: Systemic functional grammar of Spanish: A contrastive study with English, London \& New York: Continuum.

MARTIN, Jim R., 1985: Factual writing: Exploring and challenging social reality, Oxford: Oxford University Press.

Martin, Jim R., 1992: English text: System and structure, Amsterdam: John Benjamins.

Martin, Jim R. y David Rose, 2003: Working with discourse: Meaning beyond the clause, London \& New York: Continuum.

Martin, Jim R., Christian M. Matthiessen y Claire PainTER, 1997: Working with functional grammar, London: Arnold.

Matthiessen, Christian M., 1995: Lexico-grammatical cartography: English systems, Tokyo: International Language Sciences.

Montemayor-Borsinger, Ann, 2009: Tema: Una perspectiva funcional de la organización del discurso, Buenos Aires: Eudeba.

Thompson, Geoff, 2004 [1996]: Introducing functional grammar, London: Arnold.

Van Valin, Robert y Randy Lapolla, 1997: Syntax, structure, meaning and function, Cambridge: Cambridge University Press. 